\title{
Co-Prime Arrays and Difference Set Analysis
}

\author{
Usham V. Dias* and Seshan Srirangarajan*† \\ ${ }^{*}$ Department of Electrical Engineering \\ ${ }^{\dagger}$ Bharti School of Telecommunication Technology and Management \\ Indian Institute of Technology Delhi, New Delhi, India
}

\begin{abstract}
Co-prime arrays have gained in popularity as an efficient way to estimate second order statistics at the Nyquist rate from sub-Nyquist samples without any sparsity constraint. We derive an expression for the degrees of freedom and the number of consecutive values in the difference set for the prototype co-prime array. This work shows that, under the wide sense stationarity (WSS) condition, larger consecutive difference values can be achieved by using the union of all the difference sets. We provide a closed-form expression in order to determine the number of sample pairs that are available for estimating the statistics for each value of the difference set, also known as the weight function. The estimation accuracy and latency depends on the number of sample pairs used for estimating the second order statistic. We also obtain the closed-form expression for the bias of the correlogram spectral estimate. Simulation results show that the co-prime based periodogram and biased correlogram estimate are equivalent, and the reconstruction using our proposed formulation provides lower latency.
\end{abstract}

\section{INTRODUCTION}

In recent years, research has been aimed at acquiring signals at sub-Nyquist rates, pioneered by the work in the field of compressive sensing [1]-[3]. Compressive sensing relies on the fact that the signal has a sparse support in some basis set. It has found applications in many diverse fields, such as data acquisition and spectrum estimation. Sub-Nyquist sampling is essential for wideband power spectrum estimation as current hardware for analog-to-digital conversion at high rates are either difficult to design or too expensive.

All applications do not require high-fidelity reconstruction of the time-domain signal; sometimes it suffices to estimate the second order statistics of the signal. Power spectrum estimation and direction-of-arrival (DoA) estimation are some examples. Nested array is one configuration that was shown to achieve $O\left(N^{2}\right)$ degrees of freedom with just $N$ points [4]. In case of DoA estimation, $N$ is the number of sensors in the antenna array, while for power spectrum estimation $N$ is the number of samples in a given period which can generate $O\left(N^{2}\right)$ differences. The weight function for the nested array is provided in [4]. Co-prime array is an attractive alternative to the nested array [5] and uses a set of two co-prime samplers with sampling periods that are $M$ and $N$ times the Nyquist period, with $M$ and $N$ being co-prime. This configuration can resolve $O(M N)$ differences of the autocorrelation function. The weight function of the co-prime array with one of its array

Usham V. Dias is supported by a PhD fellowship from the Ministry of Electronics and Information Technology (Meity), Govt. of India. extended, is given in Table IV of [6], for a fixed value of $M$ and $N$. The general case of the weight function for any values of $M$ and $N$ has not been considered. Recently, a generalized co-prime array has been proposed [7], [8] and includes as special cases the nested array and prototype co-prime array. One of the generalized configurations is achieved by compressing the inter-element spacing of one of the sub-arrays and is referred to as the Co-prime Array with Compressed Inter-element Spacing (CACIS). The other configuration uses two displaced sub-arrays and is referred to as the Co-prime Array with Displaced Sub-arrays (CADiS). The analytical expressions for the achievable number of unique differences, maximum number of consecutive differences, and co-array aperture have been derived in [8]. Despite knowledge of the unique differences and the presence of consecutive difference values, autocorrelation estimation requires large latencies. The periodogram and correlogram based spectral estimation for the Nyquist case can be found in [9] and [10]. The bias of these spectral estimates has not been explored for the co-prime arrays or samplers.

This paper presents a generalized view of the prototype coprime array from the difference set perspective. We derive expressions for the number of sample pairs that contribute to estimating the autocorrelation at each difference value which has not been addressed in the literature so far. This expression, also known as the weight function, is important for maximizing the estimation accuracy. A closed-form expression for the bias of the co-prime based correlogram estimate is presented. We also show that larger consecutive difference values are achievable using the fact that the autocorrelation matrix is Hermitian in nature.

\section{Prototype Co-PRIME ARray}

A prototype co-prime array is shown in Fig. 1, where $M$ and $N$ are co-prime integers. The two arrays have a common element at location zero. The possible locations of the first and second array are given by $P 1$ and $P 2$ :

$$
\begin{aligned}
& P 1=\{M n d, 0 \leq n \leq N-1\} \\
& P 2=\{N m d, 0 \leq m \leq M-1\}
\end{aligned}
$$

These locations could represent the locations of an array of antennas with $d=\lambda / 2$ representing the unit inter-element spacing. From the sampling point of view, the distance $d$ represents the Nyquist sampling period $T_{s}$. Though the zeroth sample of the two samplers coincide, practically both the 


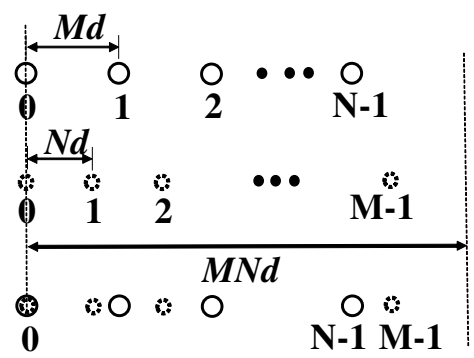

Fig. 1. General co-prime structure

samples are kept (unlike in the case of the antenna array), as it can help in synchronizing the two samplers and it maintains the uniform sampling structure of the individual samplers.

A detailed analysis of the implications of co-primality on the degrees of freedom, consecutive difference values, and the number of samples contributing to estimating the autocorrelation at each difference value holds the key to maximizing the estimation accuracy and the extent to which information can be extracted from the sub-Nyquist co-prime sampled data. If the process under consideration is WSS, then the autocorrelation matrix is Hermitian and Toeplitz. This implies that the estimate at difference value $l$ is equivalent to the conjugate of the estimate at difference value $-l$. For a real WSS process, the autocorrelation matrix is symmetric and Toeplitz.

\section{A. Co-Primality and Degrees of Freedom}

Let $x(\mathrm{Mn})$ and $x(\mathrm{Nm})$ be the outputs of the two uniform co-prime samplers. The set of self differences generated by each of these samplers, denoted by $\mathcal{L}_{S M}^{+}$and $\mathcal{L}_{S N}^{+}$, with the corresponding mirrored position sets denoted by $\mathcal{L}_{S M}^{-}$and $\mathcal{L}_{S N}^{-}$, are given below:

$$
\mathcal{L}_{S M}^{+}=\left\{l_{s} \mid l_{s}=M n\right\} \text { and } \mathcal{L}_{S N}^{+}=\left\{l_{s} \mid l_{s}=N m\right\}
$$

with $0 \leq n \leq N-1$ and $0 \leq m \leq M-1$. It is obvious that the sets $\mathcal{L}_{S M}^{+}$and $\mathcal{L}_{S N}^{+}$have $N$ and $M$ unique difference values, respectively. This also holds true for the corresponding mirrored sets $\mathcal{L}_{S M}^{-}$and $\mathcal{L}_{S N}^{-}$. The combined set of positive self difference values $\mathcal{L}_{S}^{+}$is given as (with the corresponding mirrored set denoted by $\mathcal{L}_{S}^{-}$):

$$
\mathcal{L}_{S}^{+}=\mathcal{L}_{S M}^{+} \cup \mathcal{L}_{S N}^{+}
$$

The set $\mathcal{L}_{S}^{+}$has $(M+N-1)$ unique difference values due to the fact that the difference value ' 0 ' is present in both the sets. This holds true for the corresponding mirrored set $\mathcal{L}_{S}^{-}$as well. Let $\mathcal{L}_{S}$ denote the union of all the self difference values:

$$
\mathcal{L}_{S}=\mathcal{L}_{S}^{+} \cup \mathcal{L}_{S}^{-}
$$

which contains $2(M+N-1)-1$ unique difference values. The self differences are depicted in Fig. 2. The values in the lower triangle of Fig. 2(a) belong to the set $\mathcal{L}_{S M}^{+}$while the values in the upper triangle belong to the set $\mathcal{L}_{S M}^{-}$with zero being common to both the sets. For each of the self differences, the corresponding row and column labels indicate the sample values of the acquired signal that contribute to estimating the

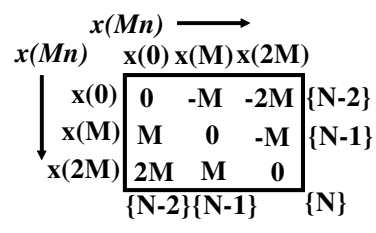

(a) $\mathcal{L}_{S M}^{+} \cup \mathcal{L}_{S M}^{-}$

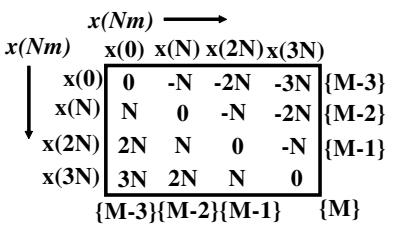

(b) $\mathcal{L}_{S N}^{+} \cup \mathcal{L}_{S N}^{-}$
Fig. 2. Self differences along with number of contributors per difference value indicated in $\{\cdot\}$ for $M=4, N=3$.

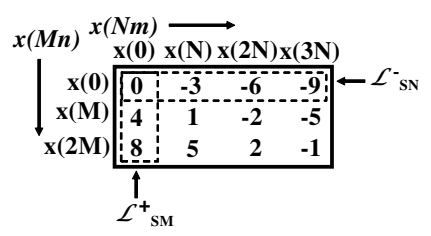

(a) Set $\mathcal{L}_{C}^{+}$

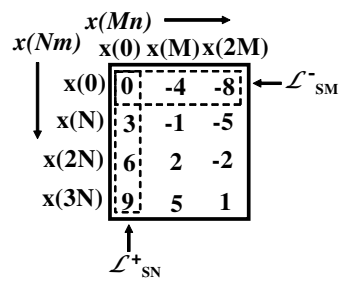

(b) Set $\mathcal{L}_{C}^{-}$
Fig. 3. Cross differences having $M N$ distinct values each appearing once for $M=4, N=3$.

value at that difference. Under the assumption of stationarity the diagonal elements represent the same difference value. Similarly, Fig. 2(b) represents the values belonging to the sets $\mathcal{L}_{S N}^{+}$and $\mathcal{L}_{S N}^{-}$with zero being common to both the sets. The cross differences generated using the two sub-arrays are given by:

$$
\mathcal{L}_{C}^{+}=\left\{l_{c} \mid l_{c}=M n-N m\right\}
$$

and $\mathcal{L}_{C}^{-}$denoting the mirrored set with $m$ and $n$ taking values as in (1). Both the cross difference sets contain $M N$ unique differences with $(M+N-1)$ self differences from $\left\{\mathcal{L}_{S M}^{+} \cup \mathcal{L}_{S N}^{-}\right\}$and $\left\{\mathcal{L}_{S M}^{-} \cup \mathcal{L}_{S N}^{+}\right\}$contained in $\mathcal{L}_{C}^{+}$and $\mathcal{L}_{C}^{-}$, respectively (and depicted in Fig. 3).

Let $\mathcal{L}_{C}$ denote the union of all the cross differences:

$$
\mathcal{L}_{C}=\mathcal{L}_{C}^{+} \cup \mathcal{L}_{C}^{-}
$$

which contains $(M N+M+N-2)$ unique differences. From Fig. 3 it can be inferred that the self differences are a subset of the cross differences.

$$
\begin{aligned}
& \mathcal{L}_{S} \subseteq \mathcal{L}_{C} \\
& \mathcal{L}_{S M}^{+} \cup \mathcal{L}_{S N}^{-} \subseteq \mathcal{L}_{C}^{+} \\
& \mathcal{L}_{S M}^{-} \cup \mathcal{L}_{S N}^{+} \subseteq \mathcal{L}_{C}^{-}
\end{aligned}
$$

The total number of unique differences achievable in the combined difference set $\mathcal{L}$, defined below:

$$
\mathcal{L}=\mathcal{L}_{C}^{+} \cup \mathcal{L}_{C}^{-} \cup \mathcal{L}_{S}^{+} \cup \mathcal{L}_{S}^{-} \text {and } \mathcal{L}=\mathcal{L}_{C}^{+} \cup \mathcal{L}_{C}^{-}
$$

is $(M N+M+N-2)$, which is the same as that in $\mathcal{L}_{C}$. Fig. 4 gives the difference values generated by the combined set $\mathcal{L}$ of the co-prime samplers. Since the zeroth sample $x(0)$ of the two samplers coincides, the value is taken only once in the combined set which is true only if the two samplers are synchronized. (For an antenna array, only one antenna 
would be placed at the common zeroth location.) This detailed analysis of the self and cross differences individually will be useful in the analysis of non-synchronized arrays [11]. A summary of the number of unique differences in each difference set is given in Table I. From Fig. 3, it is evident

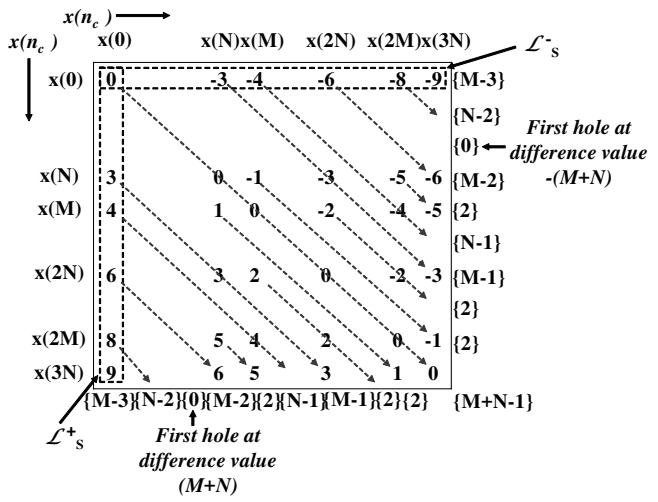

Fig. 4. Combined set $\mathcal{L}$ displaying difference values of the autocorrelation matrix with the number of contributors per difference value mentioned in $\{\cdot\}$ for $M=4, N=3$

that the negative difference values in set $\mathcal{L}_{C}^{+}$minus the self difference set, forms a subset of the flipped positive values in $\mathcal{L}_{C}^{+}$which leads to Proposition I.

\section{Proposition I:}

1) For $l_{c}$ belonging to set $\mathcal{L}_{C}^{+}-\mathcal{L}_{S}$

$$
\begin{aligned}
\left\{l_{c} \mid l_{c}<0, l_{c} \in \mathcal{L}_{C}^{+}-\mathcal{L}_{S}\right\} \subseteq\left\{-l_{c} \mid l_{c}>0, l_{c} \in \mathcal{L}_{C}^{+}\right\} \\
\subseteq\left\{-l_{c} \mid l_{c}>0, l_{c} \in \mathcal{L}_{C}^{+}-\mathcal{L}_{S}\right\}
\end{aligned}
$$

2) For $l_{c}$ belonging to set $\mathcal{L}_{C}^{-}-\mathcal{L}_{S}$

$$
\begin{aligned}
\left\{l_{c} \mid l_{c}<0, l_{c} \in \mathcal{L}_{C}^{-}-\mathcal{L}_{S}\right\} \subseteq\left\{-l_{c} \mid l_{c}>0, l_{c} \in \mathcal{L}_{C}^{-}\right\} \\
\subseteq\left\{-l_{c} \mid l_{c}>0, l_{c} \in \mathcal{L}_{C}^{-}-\mathcal{L}_{S}\right\}
\end{aligned}
$$

Proof: Let $l_{c 1}=M n_{1}-N m_{1}$ be an element of set $\mathcal{L}_{C}^{+}$where $n_{1} \in[0, N-1], m_{1} \in[0, M-1]$. Let $l_{c 2}=M n_{2}-N m_{2}$ be an element of set $\mathcal{L}_{C}^{+}$where $n_{2} \in[0, N-1], m_{2} \in[0, M-1]$. Let us assume that there exists some $l_{c 2}=-l_{c 1}$. This implies:

$$
\frac{M}{N}=\frac{m_{1}+m_{2}}{n_{1}+n_{2}}
$$

Since $M$ and $N$ are co-prime, $m_{1}+m_{2}<2 M-1$ and $n_{1}+n_{2}<$ $2 N-1$, (10) will hold if and only if $m_{1}+m_{2}=M$ and $n_{1}+n_{2}=N$.

$$
\begin{gathered}
m_{2}=M-m_{1} \\
n_{2}=N-n_{1}
\end{gathered}
$$

If $m_{1}=0$, then $m_{2}=M(\notin[0, M-1])$ but for $m_{1} \in[1, M-$ 1] we have $m_{2} \in[1, M-1]$. Similarly, if $n_{1}=0, n_{2}=N \notin$ $[0, N-1]$ but for $n_{1} \in[1, N-1]$ we have $n_{2} \in[1, N-1]$. This implies that $l_{c 2}=-l_{c 1}$ does not hold for all values of $l_{c 1} \in$ $\mathcal{L}_{C}^{+}$. Since $m_{1}=0$ gives $l_{c 1}=M n_{1}$ where $l_{c 1} \in \mathcal{L}_{S M}^{+}$and $n_{1}=0$ gives $l_{c 1}=-N m_{1}$ where $l_{c 1} \in \mathcal{L}_{S N}^{-}$, we conclude that $l_{c 1}$ and $-l_{c 1}$ exist in the set if $l_{c 1} \in \mathcal{L}_{C}^{+}-\left\{\mathcal{L}_{S M}^{+} \cup \mathcal{L}_{S N}^{-}\right\}$ or $l_{c 1} \in \mathcal{L}_{C}^{+}-\mathcal{L}_{S}$. The proof for Proposition I-2 follows along similar lines as Proposition I-1. Proposition I will enable us to prove some of the other claims made in this paper. It is also noted that Proposition 1(c) and hence Proposition 2 in [8] do not hold for the prototype co-prime array.

\section{B. Co-Primality and Continuity of Differences}

It is always desirable to have a co-prime set that creates all possible differences without any holes. This is true for the nested array, which is a special case of the generalized coprime array [8]; however this does not hold for the prototype co-prime array. It is therefore necessary to find the largest set of consecutive differences that can be generated using the co-prime pair. For the prototype co-prime array the following proposition holds.

\section{Proposition II:}

1) $\mathcal{L}_{C}^{+}$has consecutive integers in the range $-(M-1) \leq$ $l_{c} \leq N-1$

2) $\mathcal{L}_{C}^{-}$has consecutive integers in the range $-(N-1) \leq$ $l_{c} \leq M-1$

3) If $M>N, \mathcal{L}_{C}^{+}$has consecutive integers in the range $-(M+N-1) \leq l_{c} \leq-(M+1)$

4) If $M>N, \mathcal{L}_{C}^{-}$has consecutive integers in the range $(M+1) \leq l_{c} \leq(M+N-1)$

5) If $N>M, \mathcal{L}_{C}^{+}$has consecutive integers in the range $(N+1) \leq l_{c} \leq(M+N-1)$

6) If $N>M, \mathcal{L}_{C}^{-}$has consecutive integers in the range $-(M+N-1) \leq l_{c} \leq-(N+1)$

7) $\mathcal{L}$ has consecutive integers in the range $-(M+N-1) \leq$ $l_{c} \leq(M+N-1)$ which implies that this set has its first hole at $\left|l_{c}\right|=M+N$

The claims in Proposition II are depicted in Fig. 5 for $M>N$, however the proof of Proposition II is not provided here due to space constraints. It follows from this proposition that the continuous differences achievable in set $\mathcal{L}$ given by Proposition II-7 holds for both the cases when $M>N$ as well as $N>M$, and gives the maximum number of continuous difference values for a prototype co-prime array under the assumption that the process is WSS.

\section{Co-Primality and Weight function}

For autocorrelation estimation under the WSS condition, the total number of difference values that can be estimated using a co-prime sampler is equal to the cardinality of set $\mathcal{L}$ i.e. $M N+M+N-2$. The location of the first hole on either side of difference value ' 0 ' is $\pm(M+N)$. Therefore the total number of consecutive difference values possible are $2(M+N-1)+1$ in the range $[-(M+N-1),(M+N-1)]$. In addition to the ability to estimate the autocorrelation at these difference values, the accuracy of the estimation is also important. This depends on the number of data points that contribute to estimating each difference value, which is given by the weight function.

The number of elements that contribute to the estimate at each difference value is equal to the number of the elements along each diagonal with the principal diagonal giving the difference value ' 0 ', as shown in Fig. 4. The number of elements contributing to the estimation of the autocorrelation at each difference value is a key parameter in determining 
TABLE I

SUMMARY OF UNIQUE DIFFERENCES PER DIFFERENCE SET

\begin{tabular}{|c|c|c|c|c|c|c|c|c|c|c|c|}
\hline Set & $\mathcal{L}_{S M}^{+}$ & $\mathcal{L}_{S M}^{-}$ & $\mathcal{L}_{S N}^{+}$ & $\mathcal{L}_{S N}^{-}$ & $\mathcal{L}_{S}^{+}$ & $\mathcal{L}_{S}^{-}$ & $\mathcal{L}_{S}$ & $\mathcal{L}_{C}^{+}$ & $\mathcal{L}_{C}^{-}$ & $\mathcal{L}_{C}$ & $\mathcal{L}$ \\
\hline \# Unique diffs. & $N$ & $N$ & $M$ & $M$ & $M+N-1$ & $M+N-1$ & $2(M+N-1)-1$ & $M N$ & $M N$ & $M N+M+N-2$ & $M N+M+N-2$ \\
\hline
\end{tabular}

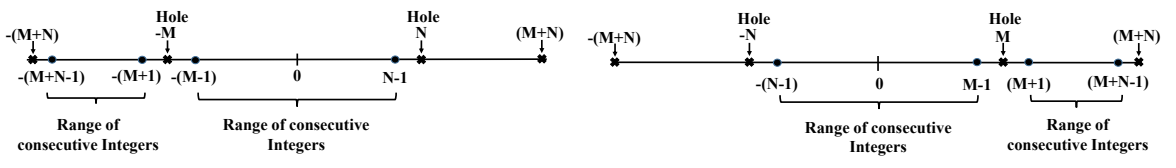

(a) Set $\mathcal{L}_{C}^{+}$when $M>N$

(b) Set $\mathcal{L}_{C}^{-}$when $M>N$

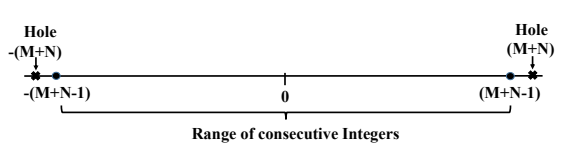

(c) Set $\mathcal{L}$

Fig. 5. Range of consecutive integers in the difference set

the estimation accuracy and minimizing the latency. For a prototype co-prime array the number of contributors at each difference value is given in Proposition III.

Proposition III: Let the number of elements contributing to the autocorrelation estimation at difference value $l$ be denoted by $z(l)$.

1) For $l$ belonging to the set $\mathcal{L}_{S M}^{+} \cup \mathcal{L}_{S M}^{-}$excluding the zeroth difference value:

$$
z(l)=(N-i),\{1 \leq i \leq N-1, l= \pm M i\}
$$

2) For $l$ belonging to the set $\mathcal{L}_{S N}^{+} \cup \mathcal{L}_{S N}^{-}$excluding the zeroth difference value:

$$
z(l)=(M-i),\{1 \leq i \leq M-1, l= \pm N i\}
$$

3) For the zeroth difference value:

$$
z(l)=M+N-1, l=0
$$

4) For $l$ belonging to the set $\mathcal{L}_{C}-\mathcal{L}_{S}$, i.e. cross differences without the self differences:

$$
z(l)=2,\left\{l \in \mathcal{L}_{C}-\mathcal{L}_{S}\right\}
$$

The first three parts of Proposition III can be inferred from Fig. 2, while the proof for Proposition III-4 is given below.

Proof of Proposition III-4: Set $\mathcal{L}_{C}^{+}$as defined in (2) and as shown in Fig. 3(a) has $M N$ possible combinations for $n \in$ $[0, N-1]$ and $m \in[0, M-1]$. This implies that only one pair $(n, m)$ contributes to each cross difference value in this set.

Let $l_{c 1}$ be an element in set $\mathcal{L}_{C}^{+}-\mathcal{L}_{S}$ and $l_{c 2}$ be an element in set $\mathcal{L}_{C}^{-}-\mathcal{L}_{S}$. From Proposition I, it follows that:

$$
\begin{gathered}
\mathcal{L}_{C}^{+}-\mathcal{L}_{S}=\left\{ \pm l_{c 1} \mid l_{c 1}>0, l_{c 1}=M n-N m\right\} \\
\mathcal{L}_{C}^{-}-\mathcal{L}_{S}=\left\{ \pm l_{c 2} \mid l_{c 2}>0, l_{c 2}=-(M n-N m)\right\}
\end{gathered}
$$

Since $\mathcal{L}_{C}^{+}$and $\mathcal{L}_{C}^{-}$are related by $l_{c 2}=-l_{c 1}$, (18) can be written as:

$$
\begin{aligned}
\mathcal{L}_{C}^{-}-\mathcal{L}_{S}=\left\{\mp l_{c 1} \mid-l_{c 1}>0, l_{c 1}=M n-N m\right\} \\
=\left\{\mp l_{c 1} \mid l_{c 1}<0, l_{c 1}=M n-N m\right\}
\end{aligned}
$$

Therefore, the sets $\mathcal{L}_{C}^{+}-\mathcal{L}_{S}$ and $\mathcal{L}_{C}^{-}-\mathcal{L}_{S}$ are equivalent and the pair $(n, m)$ that generates $l_{c 1}>0$ and $l_{c 2}>0$ are unique. This can be verified from Fig. 3(a), where difference values $l_{c 1}>0$ i.e. $\{1,2,5\}$ are generated by the $(n, m)$ pairs $\{(1,1),(2,2),(2,1)\}$, respectively, and in Fig. 3(b), where difference values $l_{c 2}>0$ i.e. $\{1,2,5\}$ are generated by the $(n, m)$ pairs $\{(2,3),(1,2),(1,3)\}$ respectively. Therefore the combined set $\mathcal{L}_{C}-\mathcal{L}_{S}$ has two contributors for each difference value which proves our claim.

\section{BIAS OF CO-PRIME CORRELOGRAM ESTIMATE}

Using Proposition III, the weight function for the prototype co-prime array can be expressed as:

$$
\begin{array}{r}
z(l)=(M+N-1) \delta(l)+\sum_{i=1}^{N-1}(N-i) \delta(|l|-M i) \\
+\sum_{j=1}^{M-1}(M-j) \delta(|l|-N j) \\
+\sum_{n=1}^{N-1} \sum_{m=1}^{M-1} 2 \delta(l-(M n-N m))
\end{array}
$$

where $-M N<l<M N$. The bias of the co-prime based correlogram is the Fourier transform of the weight function $z(l)$, whose closed-form expression is given in (21). The derivation has been omitted here due to space constraints.

$$
\begin{aligned}
W_{b}\left(e^{j \omega}\right) & =\frac{1}{s}\left\{\left|\frac{\sin \left(\frac{\omega M N}{2}\right)}{\sin \left(\frac{\omega M}{2}\right)}\right|^{2}+\left|\frac{\sin \left(\frac{\omega M N}{2}\right)}{\sin \left(\frac{\omega N}{2}\right)}\right|^{2}\right. \\
& \left.+2 \frac{\sin \left(\frac{\omega M(N-1)}{2}\right) \sin \left(\frac{\omega N(M-1)}{2}\right)}{\sin \left(\frac{\omega M}{2}\right) \sin \left(\frac{\omega N}{2}\right)}-1\right\}
\end{aligned}
$$

The scale factor $s$ can be selected as the co-prime period $M N$ or the weight at difference value ' 0 ' i.e. $M+N-1$ and is the factor used in the biased autocorrelation estimation expression.

\section{Simulation Results}

A signal model similar to that in [12], is used with sampling rate $f_{s}=1000 \mathrm{~Hz}$. The random process has three frequency bands centered at $0.1 \pi, 0.3 \pi$ and $0.6 \pi$, each with a bandwidth of $0.013 \pi$. Gaussian shaped weights are assigned to the frequency components in each band and the signal is perturbed with additive white gaussian noise $(S N R=20 \mathrm{~dB})$. The pair of co-prime samplers operate at rates which are $\frac{1}{M}$ and $\frac{1}{N}$ times the Nyquist rate with $M=4$ and $N=3$.

Let $\hat{P}_{P}$ and $\hat{P}_{C}$ denote the periodogram and the biased correlogram estimate respectively, with their definitions given 
by, $\hat{P}_{P}\left(e^{j \omega}\right)=\frac{1}{M N}\left|X_{c}\left(e^{j \omega}\right)\right|^{2}$ where $X_{c}\left(e^{j \omega}\right)$ is the Fourier transform of the signal obtained by combining the outputs of the two samplers and $\hat{P}_{C}\left(e^{j \omega}\right)=\sum_{l=-L}^{L} \hat{r}(l) e^{-j \omega l}$ with $\hat{r}(l)=\frac{1}{M N} \sum_{n_{c}} x_{c}\left(n_{c}+l\right) x_{c}\left(n_{c}\right)$ where $\hat{r}(l)$ is the biased autocorrelation estimate and $n_{c}$ is the Nyquist index location. The samples acquired in one co-prime period may not be sufficient to accurately estimate the spectrum. Hence, the estimates obtained from multiple co-prime periods are averaged to improve the accuracy of the estimate. Each coprime period is referred to as a snapshot.

The simulation results for the periodogram and correlogram co-prime spectral estimates are shown in Fig. 6 and are seen to match well. Since all the possible contributors were used as in Proposition III, reconstruction was possible with fewer snapshots. The work that we have come across in the literature has not considered the combined difference set for estimation and have chosen to multiply the outputs of the two independent samplers, thus under-utilizing the information contained in the acquired data (for e.g. (32), (8), (3), in [5], [13], [14], respectively, and [12]). An inherent bias exists as shown in Fig. 6(c), which does not vanish with more number of snapshots, and is given by (21).

The entire difference set including holes in the co-prime period were employed to estimate the spectrum without the use of a window function. The spectral estimate obtained using the entire difference set is guaranteed to be positive semi-definite. A discussion on the estimate obtained using the continuous difference set and the expression that quantifies its bias is omitted due to space constraints.

\section{CONCLUSION}

The number of elements contributing to the estimation of each difference value for the co-prime array was studied and analytical expressions have been derived. In addition, the number of unique differences and consecutive differences were studied for each of the difference sets. It has been shown that the maximum number of consecutive differences are obtained for the set which is the union of all the difference sets. This together with the expressions for the number of contributors achievable for each of the difference values can be used for improved estimation of the process autocorrelation under the WSS assumption.

The expressions for the periodogram and correlogram estimates have been provided and simulation results verify their equivalence. In addition, the closed-form expression for the bias of the correlogram estimate has been obtained. As part of the future work, we plan to provide a detailed analysis of the bias and variance of the co-prime based correlogram estimate.

\section{REFERENCES}

[1] D. Donoho, "Compressed sensing," IEEE Trans. Inf. Theory, vol. 52, no. 4, pp. 1289-1306, Apr. 2006.

[2] E. J. Candes and T. Tao, "Near-optimal signal recovery from random projections: Universal encoding strategies?" IEEE Trans. Inf. Theory, vol. 52, no. 12, pp. 5406-5425, Dec. 2006.

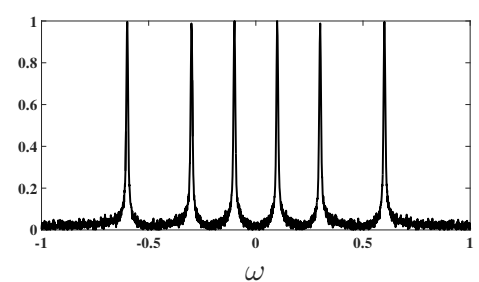

(a) True spectrum

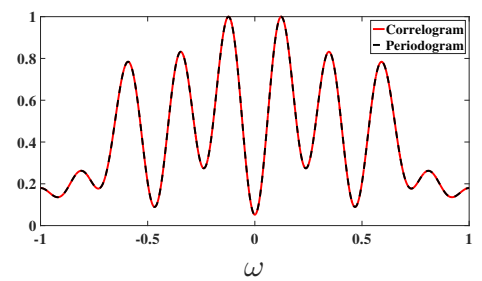

(b) Reconstructed spectrum: no. of snapshots $=2$

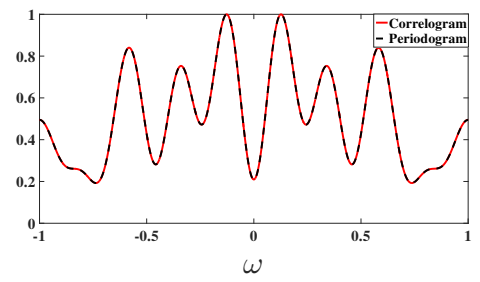

(c) Reconstructed spectrum: snapshots $=10$

Fig. 6. Simulation results for spectral estimation

[3] R. G. Baraniuk, "Compressive sensing," IEEE Signal Process. Mag., vol. 24, no. 4, pp. 118-121, Jul. 2007.

[4] P. Pal and P. P. Vaidyanathan, "Nested arrays: a novel approach to array processing with enhanced degrees of freedom," IEEE Trans. Signal Process., vol. 58, no. 8, pp. 4167-4181, Aug. 2010.

[5] P. P. Vaidyanathan and P. Pal, "Sparse sensing with co-prime samplers and arrays," IEEE Trans. Signal Process., vol. 59, no. 2, pp. 573-586, Feb. 2011.

[6] K. Han, P. Yang, and A. Nehorai, "Calibrating nested sensor arrays with model errors," IEEE Transactions on Antennas and Propagation, vol. 63, no. 11, pp. 4739-4748, Nov 2015.

[7] Q. Si, Y. D. Zhang, and M. G. Amin, "Generalized coprime array configurations," in IEEE Sensor Array and Multichannel Signal Processing Workshop (SAM). IEEE, 2014, pp. 529-532.

[8] — "Generalized coprime array configurations for direction-of-arrival estimation," IEEE Trans. Signal Process., vol. 63, no. 6, pp. 1377-1390, Mar. 2015.

[9] P. Stoica and R. L. Moses, Spectral Analysis of Signals. Upper Saddle River, New Jersey: Pearson Prentice Hall, 2005, vol. 452.

[10] E. Axell. (2011, June 29) Lecture notes on nonparametric spectral estimation. [Online]. Available: http://www.commsys.isy.liu.se/ADE/ axell-notes.pdf

[11] U. V. Dias and S. Srirangarajan, "Co-prime sampling jitter analysis," in 25th European Signal Processing Conference (EUSIPCO), 2017.

[12] P. Pal and P. P. Vaidyanathan, "Soft-thresholding for spectrum sensing with coprime samplers," in IEEE Sensor Array and Multichannel Signal Processing Workshop (SAM). IEEE, 2014, pp. 517-520.

[13] S. Ren, Z. Zeng, C. Guo, and X. Sun, "Wideband spectrum sensing based on coprime sampling," in 22nd Int. Conf. Telecommunications (ICT), 2015, pp. 348-352.

[14] A. Koochakzadeh and P. Pal, "On the robustness of co-prime sampling," in 23rd European Signal Processing Conference (EUSIPCO), 2015, pp. $2825-2829$. 